\title{
Hemi-hamate autograft arthroplasty for dorsal proximal interphalangeal fracture-dislocation: a case series of two patients
}

\author{
Mathangasinghe $\mathrm{Y}^{1}$, Rajapakse $\mathrm{YN}^{1}$, Rajapakse $\mathrm{GK}^{2}$ \\ ${ }^{1}$ Department of Anatomy, Faculty of Medicine, University of Colombo, Colombo, Sri Lanka \\ ${ }^{2}$ Department of Plastic and Reconstructive Surgery, Military Hospital, Sri Lanka
}

Keywords: Pilon fracture; arthroplasty; autograft; hamate bone; proximal phalanx

\section{Introduction}

Volar lip fractures of the base of the proximal interphalangeal joint (PIPJ) are triggered by axial loading and hyperextension injuries causing the volar plate to avulse with a ventral fragment of the base of the middle phalanx [1]. These fractures are commonly encountered during athletics and combat sports [1]. If more than $50 \%$ of the joint surface is involved, it is considered an unstable fracture [2]. Osteochondral grafts are preferably used to treat unstable fracture-dislocations [2]. Moreover, grafting is preferred in late presenters [3] compared to those who present early, where the fracture could be fixed with a higher success rate. Here, we report two cases of ventral fractures of the PIPJ managed with hemi-hamate osteochondral autografts.

\section{Case presentation}

Two male patients, aged 28 and 31 years, presented with ventral PIPJ fractures while playing basketball and following a fall in the bathroom respectively. Patients presented one week and three weeks after the injury respectively. X-rays showed comminuted unstable fracture-dislocations of the middle phalanges of the index fingers (Figure 1). Percentage involvement of the articular surfaces were more than $40 \%$. There were no concomitant injuries. Both patients were operated under general anaesthesia. The distal half of the hamate was harvested using a vertical incision (Supplementary Figure 1). A Brunner type incision was made to access the site of the fracture of the index finger. Accessory collateral ligaments were partially divided to dislocate the PIPJ. Fracture segment was removed en bloc to adequately fit the block of the hamate to achieve proper articular contour (Supplementary Figure 2). Fixation was done with two 1.3 mm titanium screws (Figure 2). An acceptable range of passive mobility was observed intraoperatively. Gentle active mobilization was started in the immediate postoperative period with a dorsal blocking splint and buddy strapping of

Correspondence: Mathangasinghe Y

E-mail: yasith@anat.cmb.ac.lk

(i) https://orcid.org/0000-0003-4641-5642

Received: 24-05-2020 Accepted: 08-10-2020

DOI: http://doi.org/10.4038/sljs.v38i3.8705

The Sri Lanka Journal of Surgery 2020; 38(3): 63-65 the index to the middle. Both had an uneventful recovery. Screws were removed six months postoperatively in the second patient to achieve further flexion. A ninety-degree flexion of the PIPJ could be achieved after eight months of surgery and the resting pain score measured by the visual analogue scale was zero.

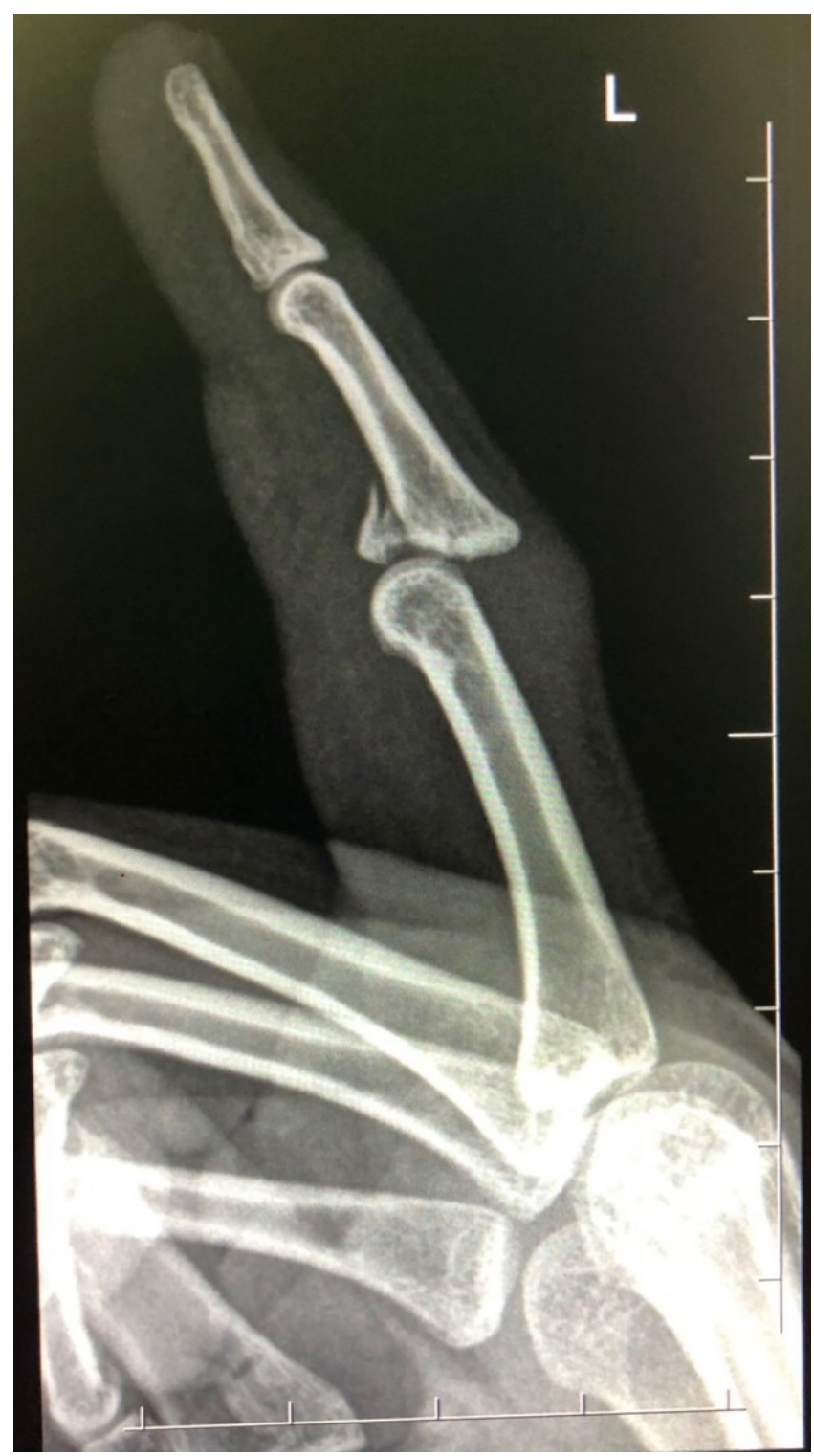

Figure 1. X-ray of the fracture dislocation of the middle phalanx of the index finger 


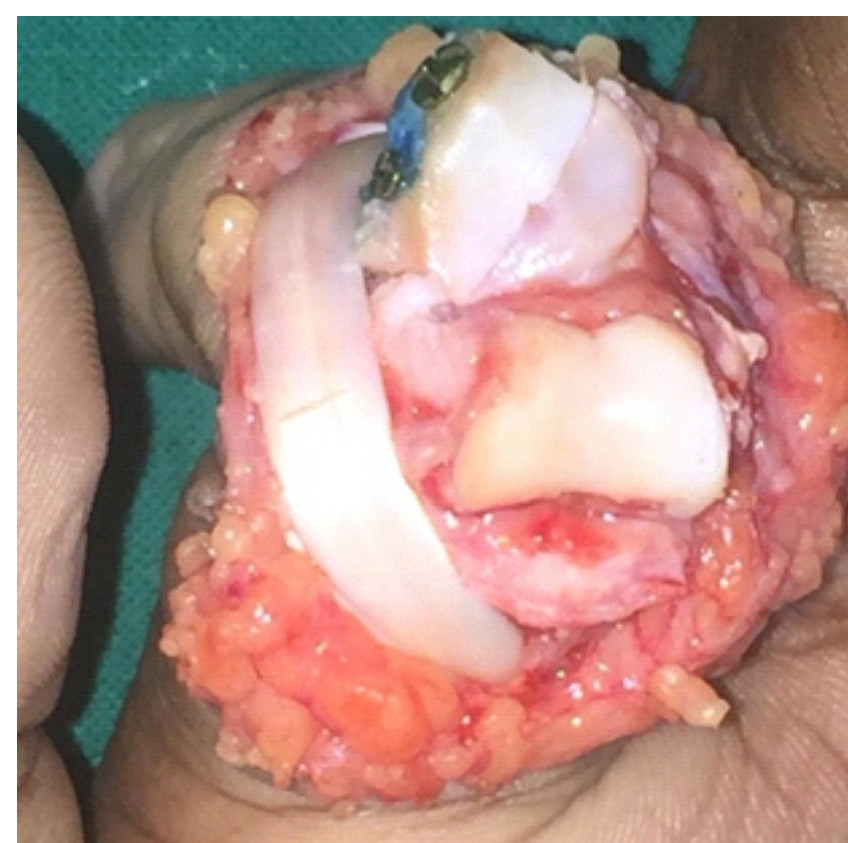

Figure 2. Fixation of the hemi hamate autograft to the middle phalanx of the index finger using titanium screws.

\section{Discussion}

Management of volar fractures of the proximal interphalangeal joints is challenging because the inability to restore the functional range of motion, dexterity, versatility and stability of the joint may compromise the activities of daily living significantly. A functioning index finger is extremely important in hand function; thus, it is essential to achieve an acceptable range of motion postoperatively.

To achieve this purpose, the treatment strategy should be personalized based on patient factors such as age, functional demand, time at presentation, comorbidities, and surgical factors such as available resources and expertise [1]. Reconstruction of the joint using autografts can be performed when a repair is not possible due to extensive comminution, late presentation and when the quality of the fractured bone fragment is poor [1].

Hemi-hamate autograft for proximal interphalangeal joint reconstruction was first described by Hasting and colleagues in 1999 [4]. The geometry of the distal hamate resembles the contours of the proximal part of the middle phalanx, therefore the distal hamate can be grafted to recreate the damaged volar buttress of the base of the middle phalanx. Soon after its original description, this surgical technique was widely adopted with minor modifications in many specialized institutions worldwide. This procedure was not performed in Sri Lanka until recently due to the sparsity of surgical expertise. We were able to perform two successful hemihamate autografts procedures in Sri Lanka as reported here.
Advantages of hemi-hamate arthroplasty include good union rates and high grip strength in long term follow up [2]. Afendras and colleagues showed a preserved range of movement of the proximal interphalangeal joint and a high level of patient satisfaction, in the majority of a follow-up cohort [5].

It is usually impossible to restore the complete range of motion of an interphalangeal joint fracture [3], however, 90 degrees of flexion is adequate to carry out most of the activities of daily living as in our case. Intra-articular screw migration, tendon irritation by screws, osteoarthritis and joint stiffness are commonly reported complications of the hemihamate arthroplasty procedure $[1,5]$, however, we observed none of these complications during eight-months follow-up of our patients. Moreover, anatomical matching of the surfaces and meticulous surgical technique to prevent damage to the articular cartilage may help in minimizing long term complications [5].

All authors disclose no conflict of interest. The study was conducted in accordance with the ethical standards of the relevant institutional or national ethics committee and the Helsinki Declaration of 1975, as revised in 2000 .

\section{References}

1. MacLean, S. and G.I. Bain, Proximal Phalangeal Joint Injuries, in Hand and Wrist Injuries In Combat Sports. 2018, Springer. p. 924. https://doi.org/10.1007/978-3-319-52902-8_2

2. Calfee, R.P., T.R. Kiefhaber, T.G. Sommerkamp, and P.J. Stern, Hemi-hamate arthroplasty provides functional reconstruction of acute and chronic proximal interphalangeal fracture-dislocations. J Hand Surg Am, 2009. 34(7): p. 1232-41. https://doi.org/10.1016/j.jhsa.2009.04.027

3. del Piñal, F., F.J. García-Bernal, J. Delgado, M. Sanmartín, and J. Regalado, Results of osteotomy, open reduction, and internal fixation for late-presenting malunited intra-articular fractures of the base of the middle phalanx. The Journal of hand surgery, 2005. 30 (5): p. 1039. e1-1039. e14. https://doi.org/10.1016/j.jhsa.2005.03.018

4. Williams, R.M., I. Hill Hastings, and T.R. Kiefhaber, PIP fracture/dislocation treatment technique: use of a hemi-hamate resurfacing arthroplasty. Techniques in hand \& upper extremity surgery, 2002. 6(4):p. 185-192. https://doi.org/10.1097/00130911-200212000-00005

5. Afendras, G., A. Abramo, A. Mrkonjic, et al., Hemi-hamate osteochondral transplantation in proximal interphalangeal dorsal fracture dislocations: a minimum 4 year follow-up in eight patients. Journal of Hand Surgery (European Volume), 2010. 35(8): p. 627-631. https://doi.org/10.1177/1753193410373311 


\section{Learning Points:}

- Volar lip fractures of the base of the proximal interphalangeal joint (PIPJ) are triggered by axial loading and hyperextension injuries.

- Osteochondral grafts are preferably used to treat unstable fracture-dislocations, especially in late presenters.

- Hemi-hamate autograft is the treatment of choice for proximal interphalangeal joint reconstruction in these fractures.

- Anatomical matching of the surfaces and meticulous surgical technique to prevent damage to the articular cartilage may help in minimizing long term complications. 